\title{
Nonalcoholic Fatty Liver Disease and the Risk of Incident Chronic Kidney Disease: A Meta-Analysis
}

\author{
Yichao Zheng ${ }^{1}$, Yifen $\mathrm{Wu}^{2}$, Yadong Lai ${ }^{1}$, Hanxu Zhuang', Guian Zheng ${ }^{3}$, * \\ ${ }^{1}$ Department of Gastroenterology, Zhangzhou Municipal Hospital of Fujian Medical University, Zhangzhou, China \\ ${ }^{2}$ Department of Hemodialysis, Zhangzhou Municipal Hospital of Fujian Medical University, Zhangzhou, China \\ ${ }^{3}$ Department of Internal Medicine, Zhangzhou Municipal Hospital of Fujian Medical University, Zhangzhou, China
}

\author{
Email address: \\ zga@fjmu.edu.cn (Guian Zheng) \\ ${ }^{*}$ Corresponding author
}

\section{To cite this article:}

Yichao Zheng, Yifen Wu, Yadong Lai, Hanxu Zhuang, Guian Zheng. Nonalcoholic Fatty Liver Disease and the Risk of Incident Chronic Kidney Disease: A Meta-Analysis. International Journal of Clinical and Experimental Medical Sciences. Vol. 4, No. 2, 2018, pp. $26-31$. doi: $10.11648 /$ j.ijcems.20180402.12

Received: April 14, 2018; Accepted: May 2, 2018; Published: May 19, 2018

\begin{abstract}
Nonalcoholic fatty liver disease (NAFLD) is a multisystem disease that affects many extrahepatic-organ systems. In this respect, several studies have suggested the possible association between NAFLD and the risk of incident chronic kidney disease (CKD). However, the extent of this association remains unclear. Thus the present meta-analysis was conducted to better characterize this association. In the meta-analysis, a comprehensive search of the databases of MEDLINE, EMBASE, Web of Science and Cochran Library was carried out from inception through September 2017. All large observational studies that investigated the association between NAFLD and the risk of incident CKD were included. The incident CKD was defined as occurrence of estimated glomerular filtration rate $(\mathrm{eGFR})<60 \mathrm{ml} / \mathrm{min} / 1.73 \mathrm{~m}^{2}$ and/or overt proteinuria. The pooled hazard ratio (HR) with $95 \%$ confidence intervals (CIs) was calculated using the random-effects model. Data were extracted from 5 large observational studies involving 57,341 adult individuals (41.3\% with NAFLD) with 1,886 cases of incident CKD. NAFLD is significantly associated with increased risk of incident CKD (HR 1.40, 95\%CI 1.22-1.61). The statistical heterogeneity of included studies was low, with an $I^{2}=25.4 \%$. In conclusion, the present meta-analysis confirms the association between NAFLD and the risk of incident $\mathrm{CKD}$.
\end{abstract}

Keywords: Nonalcoholic Fatty Liver, Chronic Kidney Disease, Meta-Analysis

\section{Introduction}

Nonalcoholic fatty liver disease (NAFLD) is one of the most commonly diagnosed liver diseases, affecting up to $33 \%$ of population worldwide [1]. Although efforts have been taken to identify and modify the risk factors of NAFLD, the incidence of NAFLD was predicted to continue to rise over the next decade [2]. It also estimated that NAFLD will become the leading cause of liver transplantation by 2030 [3]. The harm of NAFLD is not limited to liver causing nonalcoholic hepatitis, cirrhosis and liver cancer, accumulating clinical evidence indicates that the risks of developing cardiovascular and other extrahepatic diseases are increased in patients with NAFLD [3, 4]. In this respect, the possible link between NAFLD and chronic kidney disease (CKD) has drawn considerable scientific attention recently [5].

There are some evidence indicate that the presence and severity of NAFLD is associated significantly with CKD and that NAFLD predicts the development and progression of CKD independently of traditional cardiorenal risk factors [5, 6]. Although the mechanism underlying this association has not well established, experimental studies postulated that increased insulin resistance, atherogenic dyslipidemia, and the presence of proinflammatory, procoagulant, pro-oxidant, and profibrogenic mediators may be the possible explanations [3-9]. To better understand this association and estimate the risk of CKD in NAFLD patients, a meta-analysis was conducted to address this issue. 


\section{Materials and Methods}

This meta-analysis was conducted and reported according to the Meta-analysis of Observational Studies in Epidemiology statement [10].

\subsection{Search Strategy}

Two authors (Y.C. and G.A.) independently searched published studies in MEDLINE, EMBASE, Web of Science and Cochran Library from inception to September $1^{\text {st }}$, 2017, using the following search strategy without language restriction.: ((nonalcoholic fatty liver disease) OR (NAFLD) OR (nonalcoholic steatohepatitis) OR (NASH)) AND ((chronic kidney disease) OR (CKD)). References of selected retrieved articles were also manually reviewed.

\subsection{Study Selection Criteria}

The inclusion criteria for eligible studies were as follows: (i) large observational cohort studies with a sample size of at least 250 participants and a follow-up duration of at least one year; (ii) Studies evaluated the association between NAFLD and the risk of incident CKD; (iii) NAFLD was diagnosed by either abdominal imaging study or liver biopsy; (iv) incident CKD was defined as occurrence of estimated glomerular filtration rate $(\mathrm{eGFR})<60 \mathrm{ml} / \mathrm{min} / 1.73 \mathrm{~m}^{2}$ and/or overt proteinuria.

The exclusion criteria of this meta-analysis were as follows: (i) review articles, case reports, editorials, abstracts, cross-sectional studies and unpublished studies; (ii) studies ascertained NAFLD by solely liver function test without imaging or liver biopsy; (iii) studies recruited paediatric populations ( $\leq 18$ years old); (iv) Studies with data that can not be extracted.

Study eligibility was independently determined by two authors (Y.C. and G.A.). Differences in the determination of study eligibility were resolved by discussion between the authors.

\subsection{Data Extraction and Quality Assessment}

The following information was extracted from eligible articles: (i) author, year of publication, study design, study location, sample size and follow-up duration; (ii) participant characteristics (age, gender, ethnicity and comorbidities); (iii) outcome of interest; (iv) quality assessment. Quality of each study was independently evaluated by two authors (Y.C. and G.A.) using the Newcastle-Ottawa quality assessment scale that assessed each study in three areas: (i) the selection of study groups; (ii) the comparability of the groups; (iii) the ascertainment of the exposure or outcome of interest for case-control or cohort studies, respectively [11]. The author of the primary reports was contacted to request any unpublished data. If the authors did not reply, available data was used for the analyses. To ensure the accuracy, this data extraction process was independently performed by two authors noted above. Data discrepancy was resolved by referring back to the original articles.

\subsection{Statistical Analysis}

Data analysis was carried out using STATA 14.0 (Stata Corp,
College Station, Texas, USA). The adjusted hazard ratio (aHR) was used as an estimate of the relative risk to pool these data. In light of the likelihood of between-study variance because of different study designs and populations, a random-effect model rather than a fixed-effect model was used. Cochran's Q test and the $I^{2}$ statistic were used to determine the between-study heterogeneity. The heterogeneity of studies was considered insignificant if $I^{2}$ was $\leq 25 \%$, low if $I^{2}$ was > $25 \%$ and $\leq 50 \%$, moderate if $I^{2}$ was $>50 \%$ and $\leq 75 \%$ and high if $I^{2}$ was $>75 \%$ [12]. Publication bias was evaluated by visual inspection of the funnel plot or by Egger's test.

\section{Results}

\subsection{Identification of Eligible Studies}

The initial search yielded 290 articles; 279 articles were excluded on the basis of title and abstract review. A total of 11 articles underwent full-length review. Six articles were excluded (five articles have unsatisfactory exposure or outcome measures and one articles did not rely on imaging or biopsy to diagnose NAFLD). Five large observational studies were included in meta-analysis [13-17]. Figure 1 outlines the search methodology and selection process.

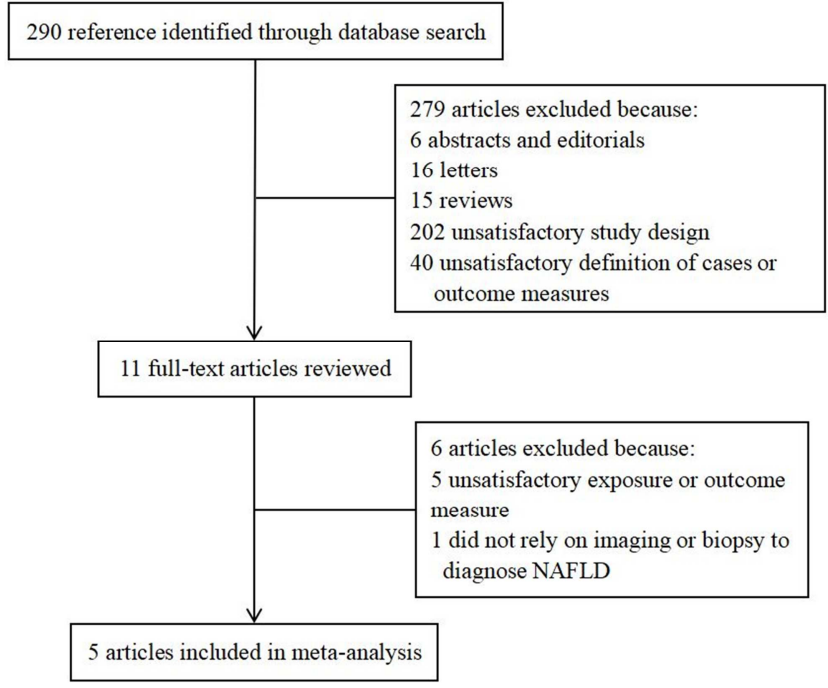

Figure 1. Flow diagram of the literature search.

\subsection{Characteristics of the Included Studies}

A total of 57,341 adult individuals with 1,886 cases of incident $\mathrm{CKD}$ were included in this meta-analysis. The medium follow-up duration was 5.2 years (inter-quartile range: 3.7-6.0 years). Among the included studies, three were prospective cohort studies $[13,14,16]$ and two were retrospective cohort studies $[15,17]$. All of the included studies diagnosed NAFLD with ultrasonography. The diagnosis of incident CKD was based on the reduced eGRF and/or overt proteinuria in all studies. The quality of the included studies ranged from six to nine. Table 1 describes the detailed characteristics and quality assessment of included studies. 
Table 1. Main characteristics of the studies included in this meta-analysis.

\begin{tabular}{|c|c|c|c|c|c|c|c|c|}
\hline References & Country & Study design & Cases & $\begin{array}{l}\text { Prevalence } \\
\text { of NAFLD }\end{array}$ & $\begin{array}{l}\text { Incidence } \\
\text { of CKD }\end{array}$ & $\begin{array}{l}\text { Follow-up } \\
\text { duration }\end{array}$ & Main results & $\begin{array}{l}\text { Quality } \\
\text { (NOS) }\end{array}$ \\
\hline Chang et al. [13] & Korea & Prospective cohort study & 8,329 & $30.2 \%$ & 324 & 3.2 years & $\begin{array}{l}\text { aHR 1.60; } \\
95 \% \text { CI 1.3-2.0 }\end{array}$ & 9 \\
\hline Targher et al. [14] & Italy & Prospective cohort study & 1,760 & $73.2 \%$ & 547 & 6.5 years & $\begin{array}{l}\text { aHR } 1.49 ; \\
95 \% \text { CI 1.1-2.2 }\end{array}$ & 9 \\
\hline Arase et al. [15] & Japan & Retrospective cohort study & 5,561 & $100 \%$ & 263 & 5.5 years & $\begin{array}{l}\text { aHR 1.35; } \\
95 \% \text { CI 1.02-1.8 }\end{array}$ & 6 \\
\hline Targher et al. [16] & Italy & Prospective cohort study & 261 & $50.2 \%$ & 61 & 5.2 years & $\begin{array}{l}\text { aHR 1.85; } \\
95 \% \text { CI 1.03-3.3 }\end{array}$ & 7 \\
\hline Sinn et al. [17] & Korea & Retrospective cohort study & 41,430 & $34.3 \%$ & 691 & 4.2 years & $\begin{array}{l}\text { aHR 1.21; } \\
\text { 95\% CI 1.03-1.44 }\end{array}$ & 8 \\
\hline
\end{tabular}

NAFLD, nonalcoholic fatty liver disease; CKD, chronic kidney disease; aHR, adjusted hazard ratio; CI, confidence interval; NOS, Newcastle Ottawa scale.

\subsection{Effect of NAFLD on the Risk of Incident CKD}

As shown in Figure 2, the forest plots indicated that NAFLD was associated with an increased risk of incident CKD (HR 1.40, 95\%CI 1.22-1.61). The statistical heterogeneity of included studies was low, with an $I^{2}=25.4 \%$.

\begin{tabular}{|c|c|c|}
\hline Study & & $\%$ \\
\hline ID & $\mathrm{HR}(95 \% \mathrm{Cl})$ & Weight \\
\hline Chang,2008 & $1.60(1.30,2.00)$ & 26.98 \\
\hline Targher,2008 & $1.49(1.10,2.20)$ & 13.18 \\
\hline Arase,2011 & $1.35(1.02,1.80)$ & 18.15 \\
\hline Targher,2014 & $\rightarrow 1.85(1.03,3.30)$ & 5.23 \\
\hline Sinn, 2017 & $1.21(1.03,1.44)$ & 36.46 \\
\hline Overall (I-squared $=25.4 \%, p=0.252)$ & $1.40(1.22,1.61)$ & 100.00 \\
\hline NOTE: Weights are from random effects analysis & & \\
\hline .303 & 3.3 & \\
\hline
\end{tabular}

Figure 2. Forest plot of the included studies assessing the association between NAFLD and incident CKD. A diamond data marker represents the overall hazard ratio and $95 \%$ CI for the outcome of interest. NAFLD, nonalcoholic fatty liver disease; CKD, chronic kidney disease; aHR, adjusted hazard ratio; CI, confidence interval.

\subsection{Evaluation of Publication Bias}

The results of visual inspection of the funnel plot revealed no evidence of publication bias (Figure 3).
Because the between-study heterogeneity was low, the sensitivity analysis was not performed. Also, a subgroup analysis was not performed as the number of studies included in this meta-analysis was small. 


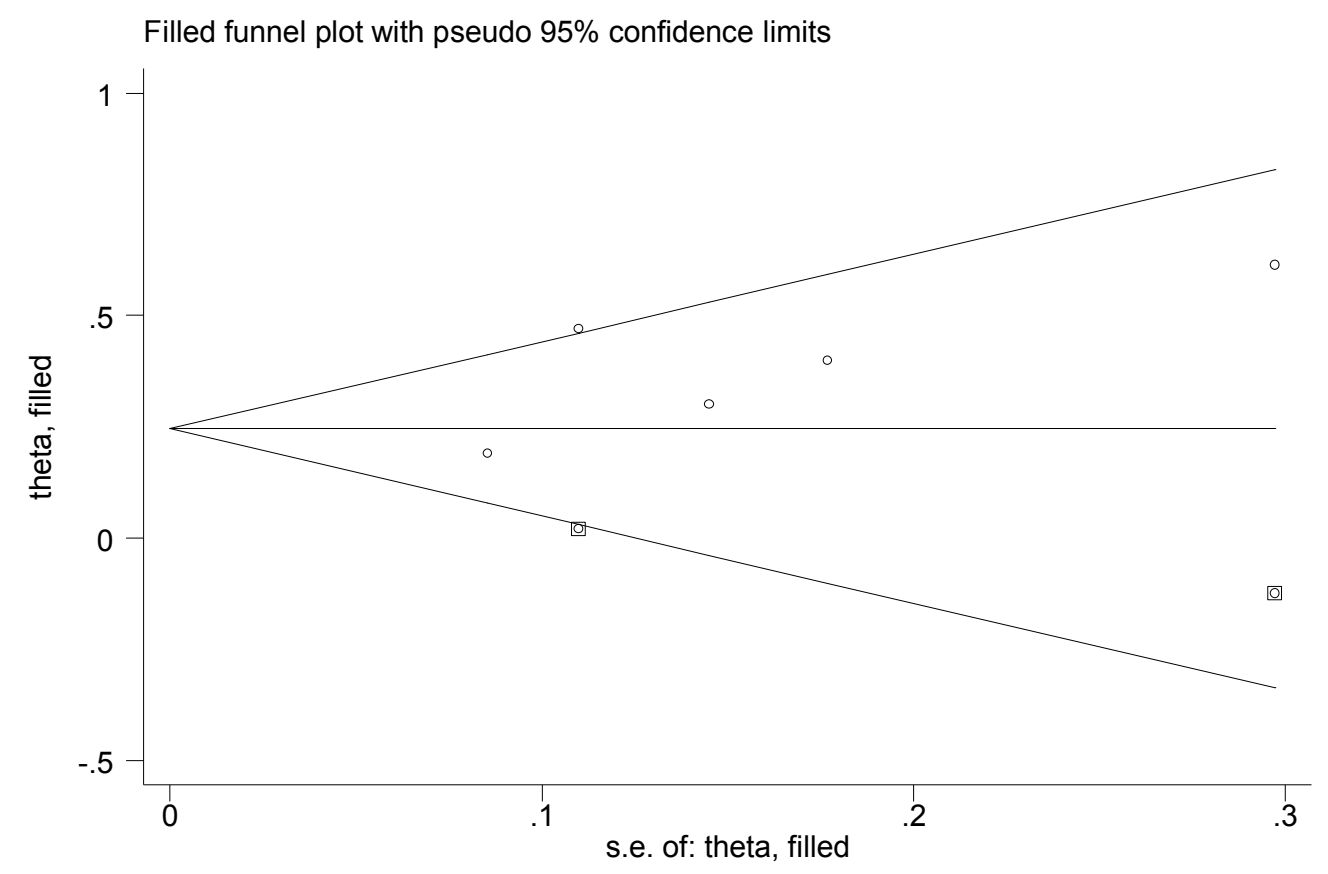

Figure 3. Funnel plot with pseudo $95 \%$ confidence limits for the analysis of NAFLD and the risk of incident CKD.

\section{Discussion}

This study is a meta-analysis to assess the association between NAFLD and the risk of incident CKD. It provides evidence of significantly increased risk of incident CKD in patients with NAFLD. In five large observational cohort studies included in the present meta-analysis, 1,886 new cases of incident CKD developed in 57,341 adult individuals (41.3\% with NAFLD) over a medium follow-up duration of 5.2 years [13-17]. To the best of our knowledge, this is the largest meta-analysis that investigate the prognostic impact of NAFLD per se on the risk of incident CKD.

Growing evidence has suggested that NAFLD is a multisystem disease that affects many extrahepatic-organ systems including the heart, vascular system and kidney [3-6, 9]. To date, many studies have demonstrated the possible link between NAFLD and the risk of CKD independent of other traditional cardiovascular risk factors [13-18]. Patients with more severe NAFLD as determined by biochemistry abnormality or increased fibrosis were also more likely to develop CKD [18, 19]. The results of the present meta-analysis are in line with these findings and better characterize the effect of NAFLD on an increased risk of incident CKD. Moreover, the findings of present meta-analysis confirm the results and extend the sample size nearly two times greater a previous meta-analysis that included both cross-sectional and longitudinal studies and used biochemistry, imaging or histology to diagnose NAFLD [19]. But it is important to note that most of the published studies that used liver biopsy to diagnose NAFLD did not have a control group and studies that used biochemistry to diagnose NAFLD lacked an acceptable diagnostic criteria, and thus may be unreasonable to be incorporated in meta-analysis.
Interestingly, the effect of NAFLD on the risk of CKD appears to be greater in the previous meta-analysis than the present one. This may be partly due to the shorter follow-up duration of included studies and smaller sample size of the previous meta-analysis. Moreover, at variance with the present meta-analysis that included exclusively large prospective cohort studies with incident cases of more advanced stage of CKD (at least stage $3 \mathrm{CKD}$ ), Musso included at least a couple of longitudinal studies that included incident cases with stages 1 or 2 CKD [19].

There are several potential factors that may underline the association of NAFLD with the risk of incident CKD. Growing evidence indicates that NAFLD, especially the nonalcoholic steatohepatitis (NASH) with varying amounts of fibrosis, exacerbates hepatic insulin resistance, promote atherogenic dyslipidemia and releases a myriad of proinflammatory, prooxidant and profibrogenic mediators that play important roles in the development of CKD and other extrahepatic complications [3-9].

Several limitations should also be acknowledged. First, NAFLD is associated with other metabolic factors including obesity, hypertension, type 2 diabetes mellitus, dyslipidemia, and insulin resistance [1, 20-24]. These confounding factors can influence the development of CKD and thus may hamper the systematic assessment of the effect of established risk factors on the outcome of interest. Since there are varying degree of confounding variables adjustment across individual studies, it was not possible to combine models in studies that adjusted for the same set of potential confounders. Second, all the included studies used ultrasonography to detect hepatic steatosis. It has limited accuracy in detecting mild steatosis as compared with liver biopsy, which is considered as the gold standard [25]. Most of published studies that used liver biopsy to diagnose NAFLD lacked a control group, and therefore 
were not included in the present meta-analysis. Third, given the lack of pathological diagnosis of NAFLD, the association between the severity of NAFLD pathology and the risk of CKD was not evaluated. Fourth, due to the limited number of included studies, the subgroup analysis was not carried out to investigate the impact of ethnicity on the outcome. Finally, the quality of included studies was not consistently high.

\section{Conclusion}

In summary, results of the largest and most comprehensive meta-analysis suggest that NAFLD is associated with a significant increased risk of CKD which is defined as eGFR < $60 \mathrm{ml} / \mathrm{min} / 1.73 \mathrm{~m}^{2}$ and/or overt proteinuria. However, the causal relationship between NAFLD and CKD is uncertain. Moreover, it remain to be defined if the risk of incident CKD is greater in NAFLD patients with advanced hepatic inflammation or fibrosis compared with those with simple steatosis. Hence, a large prospective and intervention study of patients with pathologically-defined NAFLD is required to demonstrate to the severity of NAFLD and the relative risk of incident CKD, as well as to clarify if NAFLD improvement will ultimately prevent the development of CKD or delay CKD progression.

\section{Acknowledgements}

The authors who have taken part in this study declared that they do not have anything to declare regarding funding from industry or conflict of interest with respect to this manuscript. This work was supported by Youth Research Grant of Fujian Provincial Health and Family Planning Commission (Grant 2017-1-86).

\section{References}

[1] Vernon G, Baranova A, Younossi ZM. Systematic review: the epidemiology and natural history of non-alcoholic fatty liver disease and non-alcoholic steatohepatitis in adults. Aliment Pharmacol Ther 2011; 34:274-285.

[2] Younossi ZM, Stepanova M, Afendy M, Fang Y, Younossi Y, Mir H, et al. Changes in the prevalence of the most common causes of chronic liver diseases in the United States from 1988 to 2008. Clin Gastroenterol Hepatol 2011; 9:524.e1-530.e1.

[3] Byrne CD, Targher G. NAFLD: a multisystem disease. J Hepatol 2015; 62 (Suppl):S47-S64.

[4] Adams LA, Anstee QM, Tilg H, Targher G. Non-alcoholic fatty liver disease and its relationship with cardiovascular disease and other extrahepatic diseases. Gut 2017; 66:1138-1153.

[5] Targher G, Chonchol MB, Byrne CD. CKD and nonalcoholic fatty liver disease. Am J Kidney Dis 2014; 64:638-652.

[6] Targher G, Byrne CD. Non-alcoholic fatty liver disease: an emerging driving force in chronic kidney disease. Nat Rev Nephrol 2017; 13:297-310.

[7] Petersen MC, Shulman GI. Roles of diacylglycerols and ceramides in hepatic insulin resistance. Trends Pharmacol Sci 2017; 38:649-665.

[8] Meex RCR, Watt MJ. Hepatokines: linking nonalcoholic fatty liver disease and insulin resistance. Nat Rev Endocrinol 2017; 13:509-520.

[9] Francque SM, van der Graaff D, Kwanten WJ. Non-alcoholic fatty liver disease and cardiovascular risk: pathophysiological mechanisms and implications. J Hepatol 2016; 65:425-443.

[10] Stroup DF, Berlin JA, Morton SC, Olkin I, Williamson GD, Rennie D, et al. Meta-analysis of observational studies in epidemiology: a proposal for reporting. Meta-analysis Of Observational Studies in Epidemiology (MOOSE) group. Jama 2000; 283:2008-2012.

[11] Stang A. Critical evaluation of the Newcastle-Ottawa scale for the assessment of the quality of nonrandomized studies in meta-analyses. Eur J Epidemiol 2010; 25:603-605.

[12] Higgins JP, Thompson SG, Deeks JJ, Altman DG. Measuring inconsistency in meta-analyses. BMJ 2003; 327:557-560.

[13] Chang Y, Ryu S, Sung E, Woo HY, Oh E, Cha K, et al. Nonalcoholic fatty liver disease predicts chronic kidney disease in nonhypertensive and nondiabetic Korean men. Metabolism 2008; 57:569-576.

[14] Targher G, Chonchol M, Bertolini L, Rodella S, Zenari L, Lippi $\mathrm{G}$, et al. Increased risk of CKD among type 2 diabetics with nonalcoholic fatty liver disease. J Am Soc Nephrol 2008; 19:1564-1570.

[15] Arase Y, Suzuki F, Kobayashi M, Suzuki Y, Kawamura Y, Matsumoto $\mathrm{N}$, et al. The development of chronic kidney disease in Japanese patients with non-alcoholic fatty liver disease. Intern Med 2011; 50:1081-1087.

[16] Targher G, Mantovani A, Pichiri I, Mingolla L, Cavalieri V, Mantovani W, et al. Nonalcoholic fatty liver disease is independently associated with an increased incidence of chronic kidney disease in patients with type 1 diabetes. Diabetes Care 2014; 37:1729-1736.

[17] Sinn DH, Kang D, Jang HR, Gu S, Cho SJ, Paik SW, et al. Nonalcoholic fatty liver disease and development of chronic kidney disease: a retrospective cohort study. J Hepatol 2017; 66 (Suppl): S155.

[18] Huh JH, Kim JY, Choi E, Kim JS, Chang Y, Sung KC. The fatty liver index as a predictor of incident chronic kidney disease in a 10-year prospective cohort study. PLoS One 2017; 12:e180951.

[19] Musso G, Gambino R, Tabibian JH, Ekstedt M, Kechagias S, Hamaguchi M, et al. Association of non-alcoholic fatty liver disease with chronic kidney disease: a systematic review and meta-analysis. PLoS Med 2014; 11:e1001680.

[20] Colicchio P, Tarantino G, del Genio F, Sorrentino P, Saldalamacchia G, Finelli C, et al. Non-alcoholic fatty liver disease in young adult severely obese non-diabetic patients in South Italy. Ann Nutr Metab 2005; 49:289-295.

[21] Leite NC, Salles GF, Araujo AL, Villela-Nogueira CA, Cardoso CR. Prevalence and associated factors of non-alcoholic fatty liver disease in patients with type-2 diabetes mellitus. Liver Int 2009; 29:113-119. 
[22] Assy N, Kaita K, Mymin D, Levy C, Rosser B, Minuk G. Fatty infiltration of liver in hyperlipidemic patients. Dig Dis Sci 2000; 45:1929-34.

[23] Gaggini M, Morelli M, Buzzigoli E, DeFronzo RA, Bugianesi E, Gastaldelli A. Non-alcoholic fatty liver disease (NAFLD) and its connection with insulin resistance, dyslipidemia, atherosclerosis and coronary heart disease. Nutrients 2013; 5:1544-60.

[24] European Association for the Study of the Liver (EASL), European Association for the Study of Diabetes (EASD),
European Association for the Study of Obesity (EASO). EASL-EASD-EASO Clinical Practice Guidelines for the management of non-alcoholic fatty liver disease. J Hepatol 2016; 64:1388-1402.

[25] Lee SS, Park SH. Radiologic evaluation of nonalcoholic fatty liver disease. World J Gastroenterol 2014; 20:7392-7402.

[26] Shi KQ, Tang JZ, Zhu XL, Ying L, Li DW, Gao J, et al. Controlled attenuation parameter for the detection of steatosis severity in chronic liver disease: a meta-analysis of diagnostic accuracy. J Gastroenterol Hepatol 2014; 29:1149-58. 